\title{
Experimental Validation of Amigo Based PID for a Binary Distillation Column
}

\author{
Eadala Sarath Yadav, I. Thirunavukkarasu, Ashutha K., S. Shanmugapriya
}

\begin{abstract}
Distillation is highly energy consuming process in industrial application concern. This paper focuses on energy consumption of an actuator through appropriate control design for a binary distillation column. Temperature control of binary distillation column is challenging because of existence of interaction between the variables. Independent variables of the process are fast acting (Reflux flow) and slow acting (Reboiler) with respect to process variables (Tray temperatures). Energy consumption of manipulated variable depends on efficient tuning of controller. AMIGO based PID controller design is implemented in this paper to show the optimal energy utilization of actuator. Performance analysis has been carried out to validate the control structure. It is been analyzed that based on the speed and quality requirement of the process, set of controller values can be obtained and best set of PID can be selected for real time implementation. Results depicts the efficiency of control scheme with performance index.
\end{abstract}

Keywords: Binary distillation column, AMIGO, PID, Decoupler, Interaction, FOPDT.

\section{INTRODUCTION}

$\mathrm{S}_{\text {ystems are composed with set of different components. }}$ Single input and single output (SISO) or single variable systems are compact and simple to design. But in most of the practical cases there exist multivariable controls, where there exist more than one input and output. In general change in any input will influences the dynamic change in all outputs of multivariable system. Approximate M constrained Integral Gain Optimization (AMIGO) has a set of equation to calculate the parameters of PID which are the function of $\mathrm{a}_{\mathrm{i}}$. Different studies have been made to address the problem of adjusting PID controllers in order to increase the efficiency and minimize the effect of disturbances. Although simulation results run effectively with conventional PID, while implementing in real time, they may fail to control effectively. Since there exist different constraints while executing in real time, optimized control strategy is required to overcome those constraints. Actuator saturation is one of the main constraint to overcome in real time process. The control input to the

Revised Manuscript Received on December 05, 2019

* Correspondence Author

Eadala Sarath Yadav, Department of Instrumentation and Control Engineering, Manipal Institute of Technology, Manipal Academy of Higher Education, Manipal, India. Email: sarath.eadala@gmail.com

I. Thirunavukkarasu*, Department of Instrumentation and Control Engineering, Manipal Institute of Technology, Manipal Academy of Higher Education, Manipal, India. Email: it.arasu@manipal.edu

Ashutha K., Department of Instrumentation and Control Engineering, Manipal Institute of Technology, Manipal Academy of Higher Education,

Ashutha K., Department of Chemical Engineering, Manipal Institute of Technology, Manipal Academy of Higher Education, Manipal, India. Email: shan.priya@manipal.edu Manipal, India. Email: ashuthak22@gmail.com

process should be within the region of operation. This paper deals how to select $\mathbf{M}_{\mathrm{sd}}$ values to the controller to make it work within the region of actuator saturation.

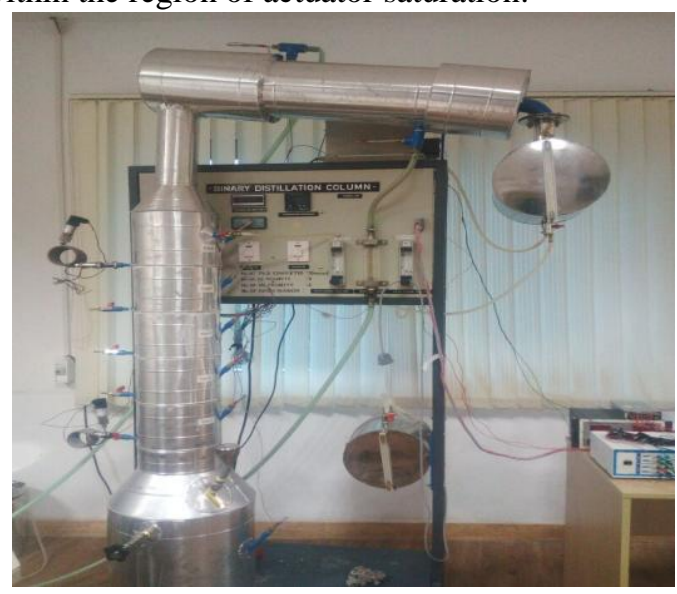

Fig.1 Real time binary distillation column

Astrom and Hagglund studied conventional controllers and it's important in the area of process control and applications, but due to the complications still emerging research carrying out in PID design [1] [2]. Several approaches are existing in the previous works about tuning PID controller for nonlinear process. PID controllers are considered by several researchers like Rivera et. al., [3], Chen and Seborg [4], Skogestad [5]. Shamsuzzoha and Lee [6] considered PID controller as broad area of research. Astrom and Hagglund suggested the methodology based on AMIGO method for tuning PID controllers [7]. There were few articles where the advanced control aspects like model predictive control is used to extract the PI/PID controller parameters [8] [9].

This paper comprises of methodology in section.II, where control design of AMIGO PID and decoupling effect has been address. Section. III shows the result analysis to validate the control design through real time binary distillation column depicted in the Fig.1, followed by conclusion in Section. IV

\section{METHODOLOGY}

\section{A. Controller Design}

Consider the TITO system with decentralized controllers $\mathrm{C}_{1}(\mathrm{~s})$ and $\mathrm{C}_{2}(\mathrm{~s})$ as shown in the fig. 2 the transfer function of the PID controllers is represented by equation (1)

$$
C_{n}(s)=K_{p n}\left(1+\frac{T_{d n} s}{1+T_{d n} s}+\frac{1}{T_{i n} s}\right)
$$

Where ' $n$ ' represents number of loops 


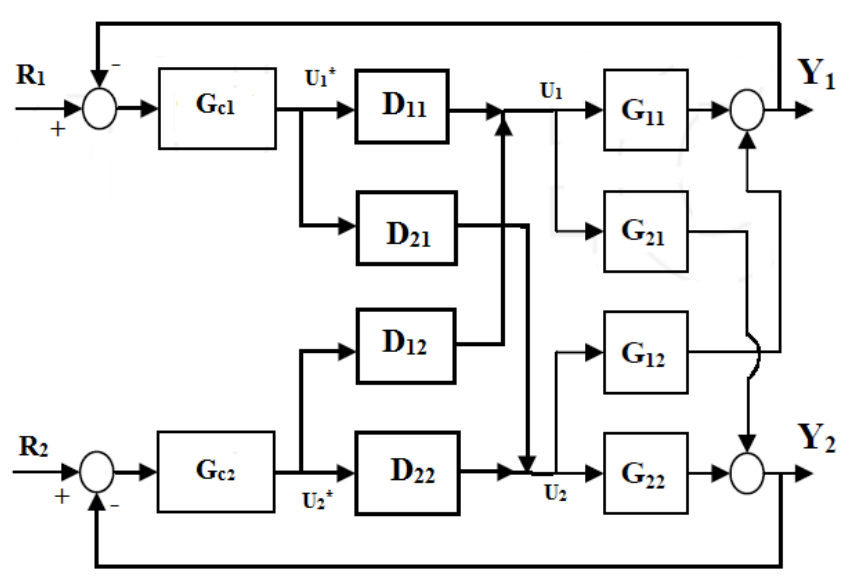

Fig.2 Decentralized control structure with de-coupler

In AMIGO methodology the controller parameter for two controllers $\mathrm{C}_{1}(\mathrm{~s})$ and $\mathrm{C}_{2}(\mathrm{~s})$ is calculated by utilizing the equation similar to that of a Ziegler- Nichols method. The sensitivity function $\mathrm{M}_{\mathrm{sd}}$ indicates the robustness of the controller design, whose range is between 1.1 and 2. This methodology is utilized in the systems whose performance is estimated by FOPTD model. The AMIGO tuning rules for PID controllers are obtained from equation (2) (3) (4). The value of $a_{i}$ used for design depends on $M_{s d}$ value, where $i=1$, $2 . . .7$ as tabulated in Table I.

$$
\begin{aligned}
& K_{p}=\frac{a_{1} t_{d}+a_{2} \tau}{k t_{d}} \\
& T_{i}=\frac{a_{3} t_{d}+a_{4} \tau}{t_{d}+a_{5} \tau} \\
& T_{d}=\frac{a_{6} t_{d} \tau}{t_{d}+a_{7} t_{d}}
\end{aligned}
$$

The parameter $a_{i}$ is determined by computing the control parameter for the process which corresponds to delay dominated, balance and lag dominated dynamics as shown in equation (5) (11) (16) respectively.

In delay dominated system the integral and derivative time is normalized by dividing them by $\mathrm{t}_{\mathrm{d}}$ and $\tau$ as in equation (6). The control parameters for delay dominated system is computed with $\mathrm{k}=1, \tau=0, \mathrm{t}_{\mathrm{d}}=1$ and is represented by equation (7) (8) (9). The controller for delay dominated system is obtained by substituting the controller parameters in equation (10).

$$
\begin{gathered}
\tau=\frac{t_{d}}{t_{d}+\tau} \\
K^{d}=\frac{1.35}{k}\left(\frac{\tau}{t_{d}}+0.185\right) \\
T_{i}^{d}=2.5 t_{d}\left(\frac{\tau+0.185 t_{d}}{\tau+0.611 t_{d}}\right) \\
T_{d}^{d}=0.37 t_{d}\left(\frac{\tau}{\tau+0.185 t_{d}}\right) \\
C^{d}(s)=K^{d}\left(1+\frac{1}{s T_{i}^{d}}+s T_{d}^{d}\right)
\end{gathered}
$$

The control parameters for balance dominated system is computed with $\mathrm{k}=1, \tau=1, \mathrm{t}_{\mathrm{d}}=1$ and is represented by equation (12) (13) (14). The controller for balance dominated system is obtained by substituting the controller parameters in equation (15).

$$
\begin{aligned}
& P^{b}(s)=\frac{1}{s+1} e^{-s} \\
& K^{b}=\frac{1}{k}\left(0.2+0.45 \frac{\tau}{t_{d}}\right) \\
& T_{i}^{b}=\frac{0.8 \tau+0.4 t_{d}}{0.1 \tau+t_{d}} \\
& T_{d}^{b}=\left(\frac{0.5 t_{d} \tau}{\tau+0.3 t_{d}}\right) \\
& C^{b}(s)=K^{b}\left(1+\frac{1}{s T_{i}^{b}}+s T_{d}^{b}\right)
\end{aligned}
$$

The control parameters for lag dominated system is computed with $\frac{k}{\tau}=1, \tau>\mathrm{td}, \mathrm{td}=1$ and is represented by equation (17) (18) (19). The controller for lag dominated system is obtained by substituting the controller parameters in equation (20).

$$
\begin{aligned}
& P^{I}(s)=\frac{1}{s} e^{-s} \\
& K^{I}=\frac{0.45}{k} \\
& T_{i}^{I}=8 t_{d} \\
& T_{d}^{b}=0.5 t_{d} \\
& C^{I}(s)=K^{I}\left(1+\frac{1}{s T_{i}^{I}}+s T_{d}^{I}\right)
\end{aligned}
$$

Table.I shows different set of $\mathrm{a}_{\mathrm{i}}$ which can be calculated by using equations (20-26).

$$
\begin{aligned}
& a_{1}=K^{b} \\
& a_{2}=K^{I} \\
& a_{3}=T_{i}^{d} \\
& a_{4}=\frac{T_{i}^{I}\left(T_{i}^{b}-T_{i}^{d}\right)}{T_{i}^{I}-T_{i}^{b}} \\
& a_{5}=\frac{\left(T_{i}^{b}-T_{i}^{d}\right)}{T_{i}^{I}-T_{i}^{b}} \\
& a_{6}=T_{d}^{d} \\
& a_{7}=\frac{T_{d}^{d}}{T_{d}^{I}}
\end{aligned}
$$

Table 1: Parameter $\alpha_{i}$ for various values of $M_{\text {sd }}$ 


\begin{tabular}{|c|c|c|c|c|c|c|c|}
\hline $\mathbf{M}_{\text {sd }}$ & $\boldsymbol{a}_{\mathbf{1}}$ & $\boldsymbol{a}_{\mathbf{2}}$ & $\boldsymbol{a}_{\mathbf{3}}$ & $\boldsymbol{a}_{\mathbf{4}}$ & $\boldsymbol{a}_{\mathbf{5}}$ & $\boldsymbol{a}_{\mathbf{6}}$ & $\boldsymbol{a}_{\mathbf{7}}$ \\
\hline 1.1 & 0.05 & 0.13 & 0.40 & 0.92 & 0.01 & 1.59 & 4.59 \\
\hline 1.2 & 0.10 & 0.26 & 0.38 & 0.93 & 0.04 & 1.62 & 4.44 \\
\hline 1.3 & 0.13 & 0.36 & 0.37 & 0.90 & 0.07 & 1.66 & 4.39 \\
\hline 1.4 & 0.16 & 0.46 & 0.36 & 0.87 & 0.11 & 1.70 & 4.37 \\
\hline 1.5 & 0.19 & 0.54 & 0.35 & 0.84 & 0.14 & 1.74 & 4.35 \\
\hline 1.6 & 0.21 & 0.61 & 0.34 & 0.82 & 0.17 & 1.78 & 4.34 \\
\hline 1.7 & 0.22 & 0.68 & 0.33 & 0.79 & 0.20 & 1.81 & 4.33 \\
\hline 1.8 & 0.24 & 0.74 & 0.32 & 0.78 & 0.23 & 1.84 & 4.32 \\
\hline 1.9 & 0.25 & 0.79 & 0.32 & 0.76 & 0.26 & 1.87 & 4.31 \\
\hline 2.0 & 0.26 & 0.84 & 0.31 & 0.75 & 0.28 & 1.89 & 4.30 \\
\hline
\end{tabular}

\section{B. Decoupler design}

A Dynamic decoupling can be easily illustrated by using TITO system. Let us consider step change in $\mathrm{U}_{1}$, which influence $Y_{1}$ and $Y_{2}$ (because of interacting term $G_{21}$ ). The influence of $\mathrm{U}_{1}$ on $\mathrm{Y}_{2}$ can should be reduced by manipulating $\mathrm{U}_{2}$. But manipulation in $\mathrm{U}_{2}$ influence $\mathrm{Y}_{1}$ and this continues. Therefore this interaction effect keeps building in the loops. This phenomena can be controlled by introducing decoupler inside the loops. Gain of decoupler is given be factor of interaction effect in the process. Figure. 2 depicts the decentralized control structure with decoupler [10].

$$
\begin{aligned}
& {\left[\begin{array}{ll}
G_{11} & G_{12} \\
G_{21} & G_{22}
\end{array}\right]\left[\begin{array}{ll}
D_{11} & D_{12} \\
D_{21} & D_{22}
\end{array}\right]} \\
& D_{11}=\frac{G_{22} F_{1}}{G_{11} G_{22}-G_{12} G_{21}} \\
& D_{22}=\frac{G_{11} F_{2}}{G_{11} G_{22}-G_{12} G_{21}} \\
& D_{12}=-\frac{G_{12}}{G_{11}} D_{22} \\
& D_{21}=-\frac{G_{21}}{G_{22}} D_{11}
\end{aligned}
$$

$$
\text { By assuming, } \quad F_{1}=\frac{G_{11} G_{22}-G_{12} G_{211}}{G_{22}}
$$

$F_{2}=\frac{G_{11} G_{22}-G_{12} G_{211}}{G_{11}}$ Equation (28) and (29) becomes

\$'1'\$.

Therefore $\mathrm{Y}_{1}$ depends on only $\mathrm{U}_{1}$ * and similarly $\mathrm{Y} 2$ depends only on $\mathrm{U}_{2}{ }^{*}$

\section{RESULT ANALYSIS}

Model identification of binary distillation column has been carried out using regression approach and refined by model using sequential relay based identification. Model using regression approach [11] is given in the equation (32) is based on the input-output data obtained from the pilot plant distillation column. First order plus dead time (FOPDT) model estimation using sequential relay method is depicted in equations (33) and (34). K, $\tau$ and $t_{d}$ of (33) and (34) are used to design controller $\mathrm{C}_{1}(\mathrm{~s})$ and $\mathrm{C}_{2}(\mathrm{~s})$ [12].

$$
G(s)=\left[\begin{array}{cc}
\frac{-0.436 e^{-1.58 s}}{1.85 s+1} & \frac{0.035 e^{-1.16 s}}{0.262 s+1} \\
\frac{-0.096 e^{-0.989 s}}{0.198 s+1} & \frac{0.088 e^{-0.76 s}}{0.349 s+1}
\end{array}\right]
$$

\section{A. sequential relay algorithm \\ 1. Consider TITO system process.}

2. Set one loop closed with relay and enable it with step change, where other loop kept opened with reference input to 'zero'.

3. Calculate controller parameters using process response with respect to relay output.

4. Use those controller parameters in the primary loop and interchange the relay to secondary loop.

5. Calculate controller parameters in secondary loop with respect to change in relay and insert those parameters in the next step.

6. Interchange relay to primary loop again calculate the controller parameters.

7. This process continues till the controller parameters of both loop converges.

$$
\begin{aligned}
G_{11 C L} & =\frac{-0.417 e^{-0.0825 s}}{1.7 s+1} \\
G_{22 C L} & =\frac{-0.083 e^{-0.011 s}}{0.32 s+1}
\end{aligned}
$$

The controller parameters $\mathrm{K}_{\mathrm{p}}, \mathrm{K}_{\mathrm{i}}$ and $\mathrm{K}_{\mathrm{d}}$ are the function of $a_{i}$ which can be obtained from the equation (2) (3) and (4) respectively. For all values of $\mathbf{M}_{\mathrm{sd}}$ the controller parameters $K_{p}, T_{i}, T_{d}$ has been calculated. For different Sensitivity function $\mathrm{M}_{\mathrm{sd}}$ values verses $\mathrm{a}_{\mathrm{i}}$ is depicted in Table.1.

Fig.3 depicts simulation of results for different values of process variable at different $\mathrm{M}_{\mathrm{sd}}$ \} respectively. It has been shown that the robustness specification given by the maximum magnitude of the sensitivity function $\mathbf{M}_{\mathrm{sd}}$ has an important effect on the controller design. As $\mathbf{M}_{\mathrm{sd}}$ increased and consequently the effect of control design parameters over the process is not efficient. On the other hand, for small values of $\mathrm{M}_{\mathrm{sd}}$ controller values satisfies the process to operate at stable region. Different values of controller parameters for varies $\mathbf{M}_{\mathrm{sd}}$ are listed in Table II. The results are analyzed based on performance indices like ISE, IAE, ITAE and ITSE as shown in Table III. It depicts that for $\mathrm{M}_{\mathrm{sd}}=1.4$ performance of controller shows maximum efficiency this process at that particular operating region. As the process is slow with respect to time, the values of $a_{i}$ and $M_{s d}$ were good at their lower set. It may varies from process to process. 
Table 2: Controller parameters for various values of $\alpha_{i}$ and $\mathbf{M}_{\mathrm{sd}}$

\begin{tabular}{|c|c|c|c|c|c|c|}
\hline \multirow{2}{*}{$\mathbf{M}_{\mathbf{s d}}$} & \multicolumn{3}{|c|}{$\mathbf{C}_{\mathbf{1}}(\mathbf{s})$} & \multicolumn{3}{|c|}{$\mathbf{C}_{\mathbf{2}}(\mathbf{s})$} \\
\cline { 2 - 7 } & $\mathbf{K}_{\mathbf{p} \mathbf{1}}$ & $\mathbf{K}_{\mathbf{i} \mathbf{1}}$ & $\mathbf{K}_{\mathbf{d} \mathbf{1}}$ & $\mathbf{K}_{\mathbf{p} \mathbf{2}}$ & $\mathbf{K}_{\mathbf{i} 2}$ & $\mathbf{K}_{\mathbf{d} \mathbf{2}}$ \\
\hline 1.1 & -0.50 & 0.58 & 0.46 & 1.37 & 1.22 & 0.17 \\
\hline 1.2 & -0.93 & 0.60 & 0.48 & 2.53 & 1.24 & 0.18 \\
\hline 1.3 & -1.30 & 0.63 & 0.50 & 3.49 & 1.31 & 0.19 \\
\hline 1.4 & -1.62 & 0.67 & 0.51 & 4.30 & 1.37 & 0.19 \\
\hline 1.5 & -1.89 & 0.71 & 0.52 & 5.00 & 1.44 & 0.20 \\
\hline 1.6 & -2.13 & 0.74 & 0.54 & 5.61 & 1.50 & 0.20 \\
\hline 1.7 & -2.34 & 0.78 & 0.55 & 6.13 & 1.56 & 0.21 \\
\hline 1.8 & -2.54 & 0.81 & 0.56 & 6.60 & 1.62 & 0.21 \\
\hline 1.9 & -2.71 & 0.84 & 0.57 & 7.02 & 1.67 & 0.21 \\
\hline 2.0 & -2.86 & 0.86 & 0.57 & 7.38 & 1.71 & -2.86 \\
\hline
\end{tabular}

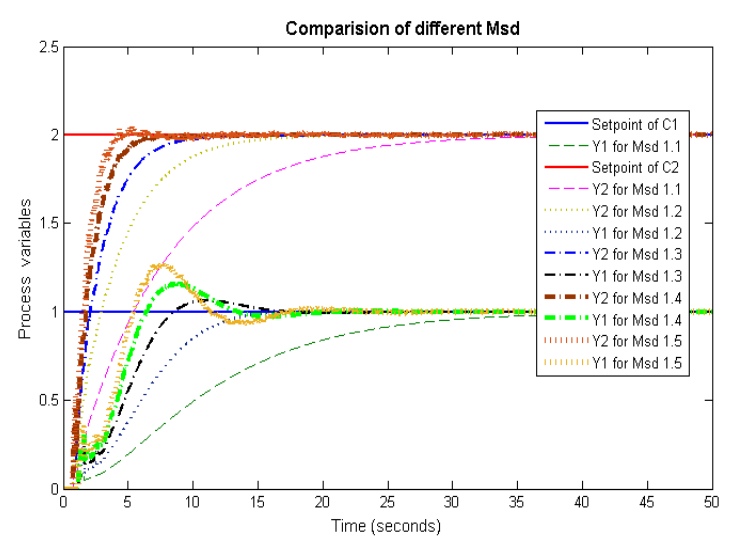

Fig.3 Simulation results for different values of process variable at different $M_{s d}$

As shown in the Fig. 3 the performance of AMIGO PID is effective for lower $M_{s d}$ values. As the $M_{s d}$ increases, the overshoot of process is gradually increasing. At $\mathrm{M}_{\mathrm{sd}}>1.6$ systems response starts oscillating because of higher gain and reaches verge and instability or may reach condition of instability at one point of time.

Table 3. Performance Indices of controllers for all $\mathbf{N}_{\mathrm{sd}}$ values for loop-1

\begin{tabular}{|c|c|c|c|c|}
\hline \multirow{2}{*}{$M_{\text {sd }}$} & \multicolumn{4}{|c|}{$C_{2}(s)$} \\
\cline { 2 - 5 } & IAE & ISE & ITAE & ITSE \\
\hline 1.1 & 7.355 & 3.98 & 49.61 & 13.63 \\
\hline 1.2 & 3.903 & 2.266 & 13.16 & 3.858 \\
\hline 1.3 & 2.696 & 1.699 & 5.848 & 1.903 \\
\hline 1.4 & 2.086 & 1.435 & 3.307 & 1.222 \\
\hline 1.5 & 1.785 & 1.293 & 2.851 & 0.9364 \\
\hline 1.6 & 10.14 & 6.535 & 356.7 & 244.4 \\
\hline
\end{tabular}
control design [13].

\begin{tabular}{|c|c|c|c|c|}
\hline \multirow{2}{*}{$\mathbf{M}_{\text {sd }}$} & \multicolumn{4}{|c|}{$\mathbf{C}_{\mathbf{1}}(\mathbf{s})$} \\
\cline { 2 - 5 } & IAE & ISE & ITAE & ITSE \\
\hline 1.1 & 10.17 & 6.193 & 80.78 & 28.11 \\
\hline 1.2 & 5.402 & 3.746 & 19.23 & 8.992 \\
\hline 1.3 & 4.298 & 2.963 & 13.53 & 5.406 \\
\hline 1.4 & 4.03 & 2.635 & 13.52 & 4.436 \\
\hline 1.5 & 4.099 & 2.508 & 15.54 & 4.43 \\
\hline 1.6 & 8.64 & 3.975 & 203 & 72.54 \\
\hline
\end{tabular}

Fig.4 depicts the closed loop response for $\mathrm{M}_{\mathrm{sd}}=1.1$. It is observed that the aggressiveness of controller $\mathrm{C}_{2}(\mathrm{~s})$ which manipulates Tray temperature- 5 is intensive thereby process variable tends to oscillates around setpoint. It is observed that controller $\mathrm{C}_{2}(\mathrm{~s})$ is reaching its maximum limits, where controller $\mathrm{C}_{1}(\mathrm{~s})$ which manipulates Tray temperature-1 has been effective over its control. Fig.5 shows the response of $M_{s d}=1.4$. It is observed when the value of $M_{s d}$ increased from 1.1 to 1.4 , the aggressiveness of controller $\mathrm{C}_{2}(\mathrm{~s})$ gradually reduced, still controller $\mathrm{C}_{1}(\mathrm{~s})$ is effectively controlling temperature at Tray-1. Actuator energy has been reduced using this control algorithm compared to the conventional

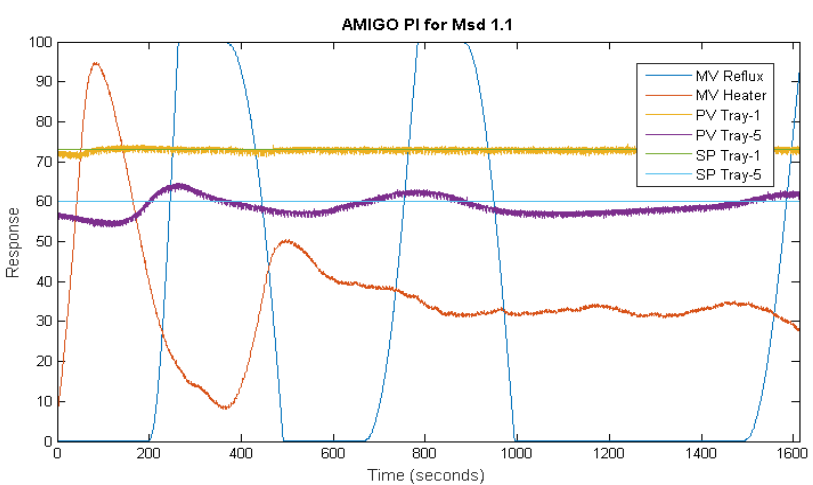

Fig.4 Real time response of AMIGO PID control $\mathrm{M}_{\mathrm{sd}}=1.1$

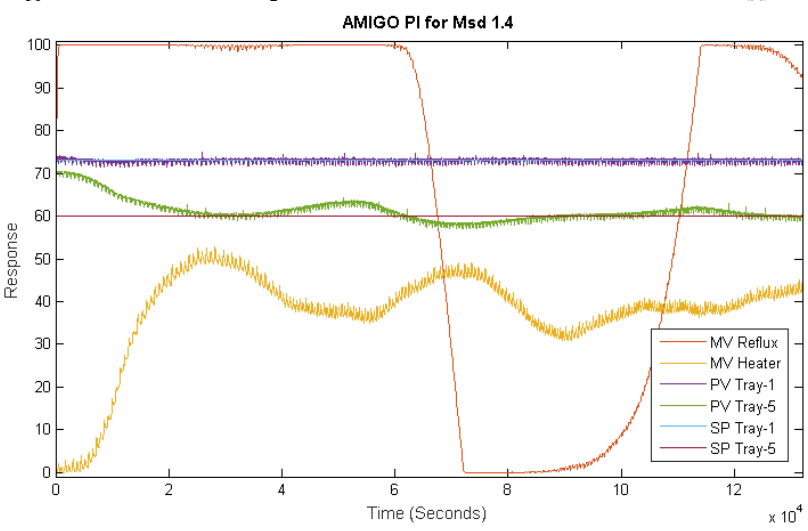

Fig.4 Real time response of AMIGO PID control $\mathrm{M}_{\mathrm{sd}}=1.4$ 


\section{CONCLUSION}

AMIGO PID is implemented to control the tray temperatures of online binary distillation process. As the process comprise of two process variables and two manipulated variables, the interaction effect is flagged as high priority to consider. As AMIGO PID can be designed using the sensitivity function $M_{\mathrm{sd}}$ which helps in selecting appropriate controller parameters depends on the process response (Slow, Medium and Fast). It can be flexible to implement for any processes by having appropriate model. Results depicts the efficiency of the algorithm.

\section{ACKNOWLEDGMENT}

Authors would like to thank Dept of Instrumentation and Control Engineering, Manipal Institute of Technology, Manipal Academy of Higher Education for providing experimental facility and first author would like to thank MAHE for providing financial support under TMA Pai scholarship scheme.

\section{REFERENCES}

1. Levine, William S. The Control Handbook (three volume set). CRC press, 2018.

2. Vrančić, Damir, et al. "Improving disturbance rejection of PID controllers by means of the magnitude optimum method." ISA transactions 49.12010, pp. 47-56.

3. Tore Hägglund, and Karl J. Astrom. Advanced PID control. Vol. 461. Research Triangle Park, NC: ISA-The Instrumentation, Systems, and Automation Society, 2006.

4. Chen, Dan, and Dale E. Seborg. "PI/PID controller design based on direct synthesis and disturbance rejection." Industrial \& engineering chemistry research 41.19 2002, pp.4807-4822.

5. Skogestad, Sigurd. "Simple analytic rules for model reduction and PID controller tuning." Journal of process control 13.4 2003, pp.291-309.

6. Shamsuzzoha, M., and Moonyong Lee. "Design of advanced PID controller for enhanced disturbance rejection of second-order processes with time delay." AIChE Journal 54.6 2008, pp.1526-1536.

7. Åström, Karl Johan, and Tore Hägglund. "Revisiting the Ziegler-Nichols step response method for PID control." Journal of process control 14.6 2004, pp.635-650.

8. Yadav, Eadala Sarath, et al. "Parameter Estimation and an Extended Predictive-Based Tuning Method for a Lab-Scale Distillation Column." ACS Omega 2019, DOI: 10.1021/acsomega.9b02713

9. Yadav, Eadala Sarath, and Thirunavukkarasu Indiran. "PRBS based model identification and GPC PID control design for MIMO Process." Materials Today: Proceedings 17 2019, pp.16-25.

10. Ravi, V. R., and T. Thyagarajan. "A decentralized PID controller for interacting nonlinear systems." 2011 International Conference on Emerging Trends in Electrical and Computer Technology. IEEE, 2011.

11. Martin, Paulo A., Darci Odloak, and Fuad Kassab. "Robust model predictive control of a pilot plant distillation column." Control Engineering Practice 21.3 2013, pp.231-241.

12. Panda, Rames C., et al. "Parameter estimation of integrating and time delay processes using single relay feedback test." ISA transactions 50.4 2011, pp.529-537.

13. Yadav, Eadala Sarath, et al. "Parameter estimation and control of a pilot plant binary distillation." Journal of Advanced Research in Dynamical and Control Systems 9.Special Issue 15 2017, pp.877-886.

\section{AUTHORS PROFILE}

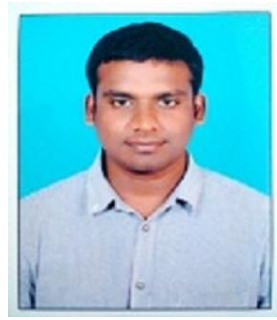

Eadala Sarath Yadav is a research scholar in ICE department, MIT, Manipal. He has 3 years of reaching experience. He worked as junior research fellow for a DST project, Govt of India His area of interest includes system modeling, advances in PID control and predictive control strategies.

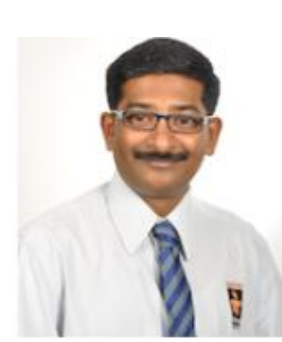

Dr. I. Thirunavukkarasu is an Associate Professor (Senior Scale) in ICE department, MIT, Manipal. He is having 12 years of teaching/research experience. His aread of interest includes robust, H-infinity control, optimal control etc. He published more than 90 research articles in conferences/journals. He organized several workshops/conferences/FDPs, He worked as principle investigator of a DST project, Govt of India. Under the excellence in teaching and research policy conference scheme of MAHE he visited 13 countries.

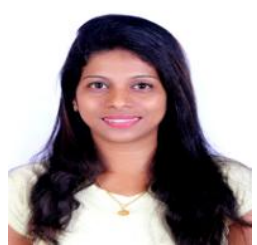

Ms. Ashutha $\mathbf{K}$ is a Senior Instrumentation Test Technology Engineer at Cummins India Limited Pune. She is having 1 years of teaching and 3 years of industrial experience. Her area of interest includes system modeling, advances in PID control, Engine electronic control strategies, and Engine Test bed control.

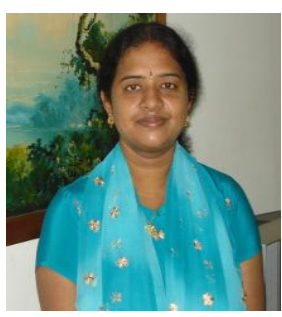

Dr. S. Shanmuga Priya is an Associate Professor (Senior Scale) in chemical Engineering department, MIT, Manipal. She is having 10 years of teaching/research experience. Her area of interest includes Energy Engineering, Heat Transfer, Environmental Engineering and Pollution Control, Environmental Studies, Safety in Chemical Industries, Cryogenic Engineering, Bioenergy engineering, Industrial waste water engineering. 